\title{
Identificação de classes de metabólitos secundários no extrato etanólico dos frutos de solanum acanthodes hook e seu potencial fungicida sobre candida albicans in vitro
}

Identification of the class of secondary metabolites of fruit extract ethanolic solanum acanthodes hook and its potential on fungicidal candida albicans in vitro

Auricélia Diogenes Gomes', Renato Abreu Lima²

'Curso de Ciências Biológicas, Faculdade São Lucas, Porto Velho, RO, Brasil

1,2Programa de Pós-Graduação - Rede BIONORTE / Universidade Federal de Rondônia, Brasil

\section{Resumo}

O presente trabalho teve como objetivo identificar as classes de metabólitos secundários do extrato etanólico dos frutos de Solanum acanthodes e avaliar a atividade fungicida sobre Candida albicans in vitro. Realizou-se a identificação de metabólitos secundários com o extrato bruto da planta e teste de cromatografia para identificar as possíveis substâncias no extrato etanólico conforme substâncias padrões isoladas de outras espécies de Solanum. Para o teste fungicida, utilizou-se a técnica de disco de fusão. No controle positivo, utilizaram-se discos

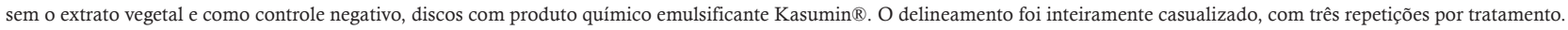
A avaliação consistiu verificar o crescimento do fungo, a cada 24 horas, durante seis dias, medindo as colônias. Os dados foram submetidos à análise de variância e as médias comparadas pelo teste de Tukey a 5\%. Com relação aos testes de reconhecimento de metabólitos secundários do extrato etanólico dos frutos de S. acanthodes, verificou-se a presença de alcaloides, glicosídeos cardiotônicos, cumarinas, taninos, triterpenos e derivados antracênicos livres. Porém, os resultados foram negativos para flavonoides. Na técnica de cromatografia foi possível identificar o glicoalcaloide Solasodina. Após 144 horas, observou que o extrato etanólico dos frutos de S. acanthodes apresentou resultados satisfatórios de C. albicans, onde ocorreu inibição do crescimento do fungo (média 2,4mm) demonstrando maior espectro inibitório se comparado como o produto químico (média 3,4mm). Conclui-se que $\mathrm{S}$. acanthodes apresenta metabólitos secundários e que estes tiveram uma relação direta na inibição de crescimento de C. albicans, porém novos métodos e concentrações precisam ser testados.

Palavras-chave: Fruta-do-lobo. Candidíase. Alcaloide.

\begin{abstract}
This study aimed to identify the classes of secondary metabolites from the ethanol extract of fruits of Solanum acanthodes and evaluate the fungicidal activity against Candida albicans in vitro. We carried out the identification of secondary metabolites with crude plant extract and chromatography test to identify potential substances in the ethanol extract as substances isolated patterns of other species of Solanum. For the test fungicide used the technique of fusion disc. For positive control, we used discs without the plant extract and as a negative control discs with emulsifying chemical Kasumin $®$. The design was completely randomized with three replicates per treatment. The evaluation consisted of checking the growth of fungus, every 24 hours for six days, measuring the colonies. Data were subjected to analysis of variance and means were compared by Tukey test at $5 \%$. Regarding the recognition of secondary metabolites from the ethanol extract of the fruits of S. acanthodes tests, the presence of alkaloids, cardiac glycosides, coumarins, tannins, triterpenes and free antracênicos derivatives was found. However, the results were negative for flavonoids. In chromatographic technique it was possible to identify the glycoalkaloid solasodine. After 144 hours, noted that the ethanol extract of the fruits of S. acanthodes showed satisfactory results of C. albicans, which occurred inhibition of fungal growth (average 2,4 mm) demonstrating greater inhibitory spectrum compared to the chemical (average $3,4 \mathrm{~mm}$ ). We conclude that $\mathrm{S}$. acanthodes presents secondary metabolites and that these had a direct relationship in the inhibition of growth of C. albicans, but new methods and concentrations need to be tested.
\end{abstract}

Keywords: Fruit-of-wolf. Candidiasis. Alkaloid. 


\section{INTRODUÇÃO}

O uso de plantas no tratamento e na cura de enfermidades e tão antigo quanto à espécie humana, tendo o conhecimento popular grande contribuição para divulgação das virtudes, porém, o uso no tratamento das doenças de plantas e controle de pragas e mais recente (LUBIAN et al., 2010). A identificação de novos compostos químicos a partir de plantas nativas ou medicinais possibilita a obtenção de substancias capazes de controlar ou inibir o desenvolvimento dos fitopatógenos (SILVA et al., 2009).

Partes da planta como raiz, caule, folhas podem fornecer substâncias ativas que serão empregadas na obtenção de um medicamento (ROSA et al., 2012). As plantas utilizadas na medicina tradicional estão sendo também cada vez mais estudadas por serem possíveis fontes de substâncias com atividades antimicrobianas frente a microrganismos prejudiciais a saúde do homem (MENDES et al., 2011).

A família Solanaceae é uma das maiores entre as Angiospermas existentes, com cerca de 2.300 espécies subordinadas a 96 gêneros. Sua distribuição geográfica está concentrada principalmente na América do Sul. No Brasil, ela apresenta grande diversidade e endemismo tendo sua ocorrência desde a Amazônia ao Sul do país, além de possuir várias espécies com atribuições ornamentais, medicinais e nutricionais (SOARES et al., 2007).

O gênero Solanum é composto por aproximadamente 1.000 a 3.700 espécies, sendo o principal gênero de Solanaceae e também um dos maiores entre as Angiospermas. Apresentam-se como plantas invasoras, ocupando os mais diversos tipos de ambientes, desde lavouras e terrenos baldios a margens de rodovias (CORDEIRO, 2008).

As plantas deste gênero também são conhecidas por produzirem uma grande variedade de saponinas (BENTO et al., 2004; ZHOU et al., 2006; NAKAMURA et al., 2008) sapogeninas (VALERI et al., 1989; WEISSENBERG, 2001); e glicoalcaloides (HALL et al., 2006; SILVA et al., 2008; DISTL et al., 2009). Estudos anteriores centraram a atenção no isolamento e caracterização da solasodina, visando sua utilização como material de partida na produção industrial de hormônios esteroidais (FIESER, 1959). Tanto que vários autores a chamam de "diosgenina do futuro" (BARBOSA-FILHO et al., 1991; BARBOSA-FILHO, 1997).

Solanum acanthodes Hook popularmente conhecida como "fruta-do-lobo", é encontrada particularmente nas regiões equatoriais. São arbustos ou arvoretas, de 2 a 5 metros de altura com cerca de $10 \mathrm{~cm}$ de diâmetro, tronco com espinhos robustos, e ramos e folhas geralmente com poucos espinhos (NEE, 2007).

Propriedades bioativas presentes em extratos vegetais e óleos essenciais, produzidos pelas plantas, como uma consequência do metabolismo secundário, mostraram-se eficientes no controle do crescimento de uma ampla variedade de microrganismos, incluindo fungos filamentosos, leveduras e bactérias, o que evidencia o potencial das plantas no combate a esses organismos patogênicos (DUARTE, 2006).

Os metabólitos secundários desempenham papeis importantes na bioquímica e fisiologia dos vegetais. Muitas classes de produtos naturais constituem grupos importantes de metabólitos secundários, estando envolvidos em muitos processos fisiológicos de plantas, particularmente naqueles que respondem ao estímulo ambiental. A composição de metabólitos secundários nos tecidos da planta pode influenciar diretamente na qualidade e toxicidade dos alimentos produzidos para humanos e animais (STOBIECKI et al., 2003). Alcaloides esteroidais são os principais metabólitos secundários encontrados no gênero e são de amplo interesse, tanto na área de ecologia como de saúde humana.

Candidíases são infecções nas quais as lesões podem ser brandas, agudas ou crônicas, superficiais ou profundas, o principal agente das candidíases é a C. albicans (AGUIAR, 2007; BARBEDO \& SGARBI, 2010).

Candida são considerados microorganismos oportunistas presentes na microbiota normal da cavidade oral e dos tratos gastrointestinal e urogenital de seres humanos. (SUZUKI, 2009). O tratamento da candidíase não tem se mostrado abrangente em sua totalidade pelo surgimento de constantes barreiras ocasionadas, e o crescente aumento de espécimes resistentes aos antifúngicos (KIRAZ \& YASEMIN, 2011; KHAN et al., 2009).

Diante das limitações de uso de antifúngicos sintéticos, evidenciadas pelo aumento da resistência pelos microrganismos, bem como pelas reações indesejadas apresentadas pelos usuários, novos agentes são propostos na tentativa de minimizar tais ocorrências. Nesse sentido, considerando a ampla atividade biológica apresentada pelos produtos de origem natural, extratos vegetais obtidos a partir do gênero Solanum têm sido investigados para determinação da atividade antimicrobiana (ARAÚJO, 
2003; SOUZA et al., 2004; LIMA et al., 2006).

Considerando essa realidade, o presente trabalho teve como objetivo identificar as classes de metabólitos secundários do extrato etanólico dos frutos de S. acanthodes e avaliar a atividade fungicida sobre C. albicans in vitro.

\section{METODOLOGIA}

Os frutos de S. acanthodes foram coletados no Sítio Nove Irmãos, Zona rural da cidade de Urupá-RO em uma área de pasto a $600 \mathrm{~m}$ do Rio Urupá. Após a coleta, os frutos foram pesados frescos, em seguida, colocados para secar em estufa a $50^{\circ} \mathrm{C}$ por 72 horas. A extração foi realizada a partir dos frutos devidamente secos e triturados, sendo colocada em Erlenmeyer contendo um litro de etanol, por sete dias, em três repetições. Posteriormente, o material foi filtrado e submetido ao processo de destilação em evaporador rotatório, resultando apenas o extrato bruto dos frutos de S. acanthodes.

\section{IDENTIFICAÇÃO DE METABÓLITOS SECUNDÁRIOS}

Foram realizados testes fitoquímicos com o extrato etanólico, baseados em precipitação e coloração dos extratos diluídos em solução e reativos específicos para cada teste conforme Radi \& Terrones (2007).

\section{CROMATOGRAFIA}

Por meio da presença ou ausência dos metabólitos secundários, procedeu-se a realização da cromatografia para identificar as possíveis substâncias no extrato etanólico dos frutos de S. acanthodes. Para isso, o extrato obtido foi analisado através da cromatografia de camada fina, utilizando placa de vidro de $10 \times 20 \mathrm{~cm}$, e como fase estacionária sílica gel GF254 $(5-40 \mu \mathrm{m})$. A placa cromatográfica foi preparada utilizando $2,0 \mathrm{~g}$ de sílica gel e água destilada, a sílica gel foi colocada em um balão pequeno de $50 \mathrm{~mL}$ e adicionado água, em seguida, o balão foi fechado e agitado durante 30 segundos aproximadamente e logo em seguida o material foi espalhado sobre a placa de vidro ate formar uma camada firme e uniforme. A placa ficou em repouso para secar a temperatura ambiente e logo foi ativada na estufa a $100^{\circ} \mathrm{C}$. Colocou-se $45 \mathrm{~mL}$ de clorofórmio e $5 \mathrm{~mL}$ de etanol para a concentração de $95,5 \%$ a ser usado dentro da câmara, e também utilizou-se $2 \mathrm{~mL}$ de clorofórmio no tubo de ensaio com Solasonidina como padrão 1 e Diogenina como padrão 2 de Solanum para comparação.

A amostra do extrato foi adicionada a placa, utilizando um tubo capilar e colocados dois pingos da amostra na placa de cromatografia de camada fina. Depois de secar a amostra, a placa é colocada dentro de uma câmara cromatográfica e deixada correr até uma distancia de $9-10 \mathrm{~cm}$, aproximadamente. Logo a placa é sacada da câmara, deixando secar a temperatura ambiente e colocada em uma câmara reveladora com iodo, para detectar manchas existentes na placa.

\section{CULTURA DO FUNGO C. ALBICANS}

No Laboratório de Microbiologia da Faculdade São Lucas, utilizou-se a técnica de disco de fusão, onde discos de cinco $\mathrm{mm}$ de diâmetro de culturas de isolados do fungo $C$. albicans (ATCC 10.231), foram colocados no centro de placas de Petri contendo meio Batata Dextrose Agar (BDA), na área periférica das placas, foram dispostos simetricamente quatro discos de papel-filtro, que foram embebidos em $1 \mathrm{~mL}$ de extrato vegetal durante 1 minuto, obtendo-se a $0,12 \mathrm{~mL}$ de extrato para cada disco. Como controle positivo, utilizou-se discos embebidos em água destilada e controle negativo, o produto químico Kasumin ${ }^{\circledR}$, ambos na concentração de $1 \mathrm{~mL}$. Após esse processo, as placas foram incubadas a $25^{\circ} \mathrm{C}$ durante seis dias. A avaliação consistiu em medir o diâmetro das colônias (média de duas medidas diametralmente opostas) iniciadas após 24 horas de incubação, perdurando os seis dias, ou seja, até o momento em que as colônias fúngicas do tratamento testemunha atingiram toda 
a superfície da placa. O delineamento estatístico utilizado foi o inteiramente casualizado, com três repetições por tratamento.

Os dados obtidos foram submetidos à análise de variância e as médias comparadas pelo teste de Tukey a $5 \%$ de probabilidade.

\section{RESULTADOS E DISCUSSÃO}

O material vegetal fresco coletado teve um rendimento de 3.586 g. Após sete dias na estufa elétrica, os frutos foram triturados, obtendo-se $576,71 \mathrm{~g}$, e a quantidade final de extrato bruto do material vegetal foi de $90 \mathrm{~mL}$.

Com relação aos testes de reconhecimento de metabólitos secundários do extrato etanólico dos frutos de S. acanthodes, verificou-se que os resultados foram positivos para alcaloides, glicosídeos cardiotônicos, cumarinas, taninos, triterpenos e derivados antracênicos livres. Porém, os resultados foram negativos para flavonoides.

Um importante fator a ser considerado quando se realiza qualquer pesquisa envolvendo plantas medicinais e se tenta extrapolar os resultados obtidos, é quanto a fatores ambientais envolvidos no momento da coleta da planta, como sazonalidade, clima, tipo de solo e temperatura do ar. De acordo com Freitas et al. (2004) a produção de metabólitos secundários pela planta ocorre em função da interação planta versus ambiente em resposta a fatores químicos e biológicos. Este fato pode explicar resultados divergentes de extratos da mesma espécie, mas coletado em locais e períodos diferentes.

Acredita-se que os metabólitos secundários presentes em $\mathrm{S}$. acanthodes possam ter algum papel na defesa das plantas e uma relação com a resistência a doenças fúngicas e com a repulsão de insetos (HUNZIKER, 2001). As plantas do gênero Solanum são conhecidas por possuírem em sua composição química principalmente alcaloides esterodais, além de uma grande variedade de saponinas, sapogeninas, flavonoides e glicoalcaloides (VAZ, 2010) e esses metabólitos secundários são descritos na literatura por possuírem potencial biológico contra microrganismos.

Os glicoalcaloides já foram detectados em mais de 350 espécies de plantas, a maioria em Solanum spp. Ou outros membros da família Solanaceae. São conhecidas mais de 75 estruturas de agliconas (alcaminas) de ocorrência natural, e esqueleto colestano (C27) contendo nitrogênio em anéis ou em grupos que lhes confiram basicidade e alguma atividade biológica (SILVA et al., 2005).

Em estudo fitoquímico com o caule de S. acanthodes, Pires et al. (2009) utilizando reagentes específicos para reconhecimento de alcaloides, glicosídeos cardiotônicos, de cumarinas voláteis, flavonoides, taninos saponinas, triterpenos e derivados antracênicos livres, verificaram que os resultados obtidos foram positivos para alcaloides, triterpenos, flavonoides e saponinas. Além disso, foram encontrados glicosídeos cardiotônicos, usando somente o reagente de Keller-Killiani. Só não foram encontrados derivados antracênicos livres, taninos e cumarinas voláteis. Com a identificação dos componentes presentes no extrato etanólico das inflorescências utilizando reagentes específicos, conclui-se que o mesmo apresenta metabólitos secundários, que são compostos de grande interesse na medicina tradicional.

Resultados semelhantes foram encontrado por Alves et al. (2005) ao testar extratos brutos etanólicos das folhas e frutos verdes e maduros de S. palinacanthum, observando uma inibição do crescimento da levedura C. albicans. Além disso, Nascimento et al. (2006) relataram a atividade antimicrobiana in vitro de extratos vegetais de S. paniculatum Lam., sobre o crescimento da bactéria Ralstonia solanacearum.

O extrato etanólico do fruto de S. acanthodes analisado utilizando a cromatografia de camada fina que obtiveram algumas manchas e foram calculados valores de $\mathrm{Rf}$, observando os seguintes resultados: Solasodina - 5,5 $\div 9=0,61$; Diogenina - não foi encontrado na espécie; Cálculo de Rf: extrato $=5,5 \div 9=0,61$.

De acordo com o estudo cromatográfico de camada fina com o extrato do fruto de $S$. acanthodes utilizando-se de Solasodina e Diogenina isoladas de outras espécies de Solanum utilizando como padrão, pode-se verificar a presença de uma das estruturas no extrato analisado onde o calculo de Rf apresentou o valor de 0,61 , no qual o extrato se mostrou positivo para Solasodina e negativo para Diogenina.

Utilizando também a técnica de cromatografia de coluna fina, Rigo (2008) detectou a presença de 17 manchas diferentes e, utilizando padrões obtidos de outras espécies do gênero Solanum, identifi- 
cou a presença dos compostos químicos no extrato dos frutos: Solasodina, Solasodieno, Clorogenina, Tomatidenol, Tigogenina e Diogenina.

Atualmente, inúmeros experimentos evidenciam o fato de que muitos metabólitos secundários presentes nas plantas, como os terpenos, alcaloides, glicosídeos cianogênicos, saponinas, taninos e antraquinonas são aleloquímicos que representam caracteres adaptativos e que tem se diversificado durante a evolução pela seleção natural a fim de proteger as plantas contra vírus, bactérias, fungos, plantas concorrentes e contra os herbívoros (WINK, 2003).

Com relação ao potencial fungicida, verificou-se que o extrato etanólico dos frutos de S. acanthodes apresentou inibição sobre C. albicans, notando-se que no final de 144 horas, a média de inibição das colônias dos fungos utilizando o extrato vegetal foi de $2,4 \mathrm{~mm}$; no controle positivo, utilizando a água destilada estéril, a média foi de $4,6 \mathrm{~mm}$, enquanto que no controle negativo, utilizando o produto químico, a inibição média foi de 3,4 mm (Figura 1).

\begin{tabular}{llllllll}
\hline Tratamentos & \multicolumn{7}{c}{ Horas } \\
\cline { 2 - 8 } & 24 & 48 & 72 & 96 & 120 & 144 & Médias* $^{*}$ \\
\cline { 2 - 8 } $\begin{array}{l}\text { Extrato vegetal } \\
\text { Produto }\end{array}$ & $1,0 \mathrm{a}$ & $1,2^{\mathrm{a}}$ & $1,4 \mathrm{a}$ & $1,7 \mathrm{a}$ & $2,1 \mathrm{aB}$ & $2,4 \mathrm{aB}$ & $1,63 \mathrm{a}$ \\
químico & $1,2^{\mathrm{a}}$ & $1,6^{\mathrm{a}}$ & $1,9 \mathrm{a}$ & $2,4 \mathrm{aB}$ & $2,9 \mathrm{aB}$ & $3,4 \mathrm{aC}$ & $2,23 \mathrm{Ab}$ \\
Água destilada & $1,5^{\mathrm{a}}$ & $2,4 \mathrm{aB}$ & $2,7 \mathrm{aB}$ & $3,3 \mathrm{aC}$ & $3,7 \mathrm{aC}$ & $4,6 \mathrm{C}$ & $3,03 \mathrm{Ac}$ \\
Médias & $1,23^{\mathrm{a}}$ & $1,73 \mathrm{a}$ & $2,00 \mathrm{a}$ & $2,46 \mathrm{aB}$ & $2,9 \mathrm{aB}$ & $3,46 \mathrm{aC}$ & $2,29 \mathrm{aB}$ \\
\hline
\end{tabular}

*Médias seguidas de mesma letra maiúscula, na coluna, e mesma letra minúscula, na linha, não diferem entre si pelo teste de Tukey $(\mathrm{p} \leq 0,05)$.

Figura 1. Média (mm) de inibição de crescimento do fungo C. albicans submetidos à exposição do produto químico, água destilada e extrato vegetal dos frutos de S. acanthodes in vitro durante 144 horas.

Muitas espécies de fungos não albicans mais comumente isoladas são menos susceptíveis aos derivados azólicos, dificultando o tratamento dessas infecções. Embora a susceptibilidade das leveduras do gênero Candida aos antifúngicos disponíveis seja variável e previsível, nem sempre uma determinada amostra isolada segue o padrão geral; essa é uma das razões da crescente importância dos testes de susceptibilidade (CROCCO et al., 2004).

Bento et al. (2004) fez uma investigação fitoquímica e farmacológica do extrato etanólico obtido dos frutos de S. acanthodes coletados no município de Porto Velho-RO para avaliar as atividades antinociceptiva em camundongos. Os animais receberam injeção intraplantar de glutamato e nocicepção induzida pela formalina, passando a lamber a pata, como indicativo de dor. Estes animais foram tratados com o extrato etanólico dos frutos dessa espécie, onde se observou de forma significativa a redução de lambida ou mordida induzidas pelo glutamato e formalina, sendo, portanto, considerado antinoceptivo e expressivo na dor inflamatória.

Azevedo et al. (2004) avaliaram o potencial antioxidante do extrato etanólico do fruto de S. acanthodes e S. crinitum. Onde verificaram que os resultados mostraram que os dois extratos foram eficazes contra o agente oxidante DPPH (2,2-difenil-1-picrilidrazila), utilizando-se o método do radical livre estável. Contudo, o extrato do $\mathrm{S}$. acanthodes mostrou-se dependente da dose, pois com o aumento da dose utilizada houve um aumento da atividade antioxidante, ao contrário do extrato do S. crinitum, que se mostrou estável.

Santos \& Lima (2013), em estudo com o extrato etanólico das folhas de S. acanthodes, verificou que a planta apresentou efeito de inibição sobre C. albicans, apresentando maior índice de inibição utilizando o extrato vegetal foi de 2,4 em comparação ao produto químico que foi de 3,4 . O mesmo evidencia-se ao estudo comparado ao de Neto \& Lima (2014) ao verificar que o extrato etanólico dos frutos de Solanum grandiflorum, apresentou potencial fungicida sobre Rhizoctonia solani, notando-se que no final do experimento, a média de inibição das colônias dos fungos utilizando o extrato vegetal foi de 4,4; no controle positivo, utilizando a água destilada estéril, a média foi de 5,6, enquanto que no controle negativo, utilizando o produto químico, a inibição média foi de 4,5.

Estudos realizados por Anselmo \& Lima (2014) também foi positivo quando utilizado extrato 
vegetal do gênero Solanum contra $C$. albicans, possivelmente devido à presença de um ou combinação de vários metabólitos secundários. Resultados semelhantes foram encontrado por Alves et al., (2005) ao testar extratos brutos etanólicos das folhas e frutos verdes e maduros de S. palinacanthum, observando uma inibição do crescimento da levedura C. albicans. Além disso, Nascimento et al., (2006) relataram a atividade antimicrobiana in vitro de extratos vegetais de S. paniculatum Lam., sobre o crescimento da bactéria Ralstonia solanacearum.

\section{CONCLUSÕES}

Conclui-se que S. acanthodes é de grande importância na medicina, apresentando metabólitos secundários que servem de matéria-prima para diferentes fármacos e afecções e indicações terapêuticas a serem utilizadas e testadas e que a mesma apresentou atividade fungicida sobre C. albicans, pois apresentou inibição de crescimento se comparado ao produto químico, porém novos métodos e concentrações precisam ser testados.

\section{AGRADECIMENTOS}

Ao Laboratório de Fitoquímica da Faculdade São Lucas pelo auxílio na produção dos extratos e ao Laboratório de Microbiologia pela cultura dos fungos e a FAPEAM pela concessão de bolsa de pesquisa.

\section{REFERÊNCIAS}

AGUIAR, M. M. G. B. Desenvolvimento de Novos Comprimidos Bucais de Nistatina para o Tratamento de Candidíase Oral. 2007. 146f. Dissertação (Mestrado em Ciências Farmacêuticas) - Faculdade de Farmácia, Universidade Federal do Rio de Janeiro, Rio de Janeiro, 2007.

ALVES, A. A.; PIRES, A. F.; LINARDI, V. R.; REINA L. C. B.; GALVÃO, C. Atividade Antibacteriana e Antifúngica dos extratos brutos etanólicos de Solanum palinacanthum. In: ENCONTRO DE PESQUISA DE IES DO SISTEMA ESTADUAL DE MINAS GERAIS, 3, Anais...Caratinga: MG, 2005.p.143.

ANSELMO, J.S.; LIMA, R.A. Identificação de metabólitos secundários no extrato etanólico das folhas de Solanum jamaicese (Solanaceae) e seu potencial fungicida sobre Candida albicans in vitro. Revista Eletrônica de Farmácia, v.11, n.1, p.11-20, 2014.

ARAÚJO, J.C.L.V. Perfil de sensibilidade de microrganismos oportunistas de origem clínica e ambiental a óleos essenciais. 2003.77p. Dissertação (Mestrado em Produtos Naturais e Sintéticos Bioativos) - Departamento de Ciências Farmacêuticas, Universidade Federal da Paraíba, João Pessoa.

AZEVEDO, M.S.; VALE, C.A.S.; SANTOS, O.A.; SOARES, P. Atividade larvicida de frutos de Solanum stramonifolium contra Anopheles darlingi. In: Simpósio de Plantas Medicinais, 18, Anais... Manaus: AM, 2004. p.119.

BARBEDO, L. S.; SGARBI, D. B. G. Candidíase. Jornal Brasileiro de Doenças Sexualmente Transmissíveis, v.22, n.1, p.22-38, 2010.

BARBOSA-FILHO, J.M.; AGRA, M.F.; OLIVEIRA, R.A.G.; PAULO, M.Q.; TROLIN, G.; CUNHA, E.V.L.; ATAIDE, J.R.; BHATTACHARYYA, J. Chemical and Pharmacological Investigation of Solanum Species of Brazil - A Search for Solasodine and Other Potentially Useful Therapeutic Agents. Memórias do Instituto Oswaldo Cruz, v.86, n.2, p.189-191, 1991. 
BARBOSA-FILHO, J.M. Quimiodiversidade e Potencialidade Farmacológica da Flora Paraibana. Caderno de Farmácia, v.13, n.2, p.85-102, 1997.

BENTO, A.F.; AZEVEDO, M.S.; LUIZ, A.P.; MOURA, J.A.; SANTOS, A.R.S. Atividade antinoceptiva do extrato etanólico do fruto de Solanum acanthodes Hook.f. em camundongos. Revista Brasileira de Farmacognosia, v.14, n.1, p.9-10, 2004.

CORDEIRO, L.N. Efeito in vitro de extratos etanólicos da raiz de jurubeba (Solanum paniculatim L.) e das folhas de Melão-de-São-Caetano (Momordica charantia L.) sobre ovos e larvas de nematoides gastrintestinais de caprinos. (Dissertação de Mestrado em Zootecnia). Patos, PB: UFCG, 2008. 64 p.

CROCCO, E.I.; MIMICA, L.M.J.; MURAMATU L.H.; GARCIA,C.; SOUZA, V.M.; RUIZ L.R.B.; ZAITZ, C. Identificação de espécies de Candida e susceptibilidade antifúngica in vitro: estudo de 100 pacientes com candidíases superficiais. Anais Brasileiros de Dermatologia, v.79, n.1, p.689-697, 2004.

DISTL, M.; WINK, M. Identification and Quantification of Steroidal Alkaloids from Wild Tuber-Bearing Solanum species by HPLC and LC-ESI-MS. Potato Research, v.52, n.1, p.79-104, 2009.

DUARTE M.C.T. Atividade Antimicrobiana de Plantas medicinais e aromáticas utilizadas no Brasil. Construindo a história dos Produtos Naturais. Multi Ciência: Revista Interdisciplinar dos Centros e Núcleos da Unicamp, v.7 n.1, p.1-16, 2006.

FIESER, L.F.; FIESER M. Steroids: Reinhold Publishing Corporation. New York - Chapman \& Hall, LTD. London. 1959.

FREITAS, M.S.M.; SOUZA, P.H.; BELLO, O.I.; JAQUES, R.S. Crescimento e produção de fenóis totais em carqueja [Baccharis trimera (Less.) D.C.] em resposta à inoculação com fungos micorrízicos arbusculares, na presença e na ausência de adubação mineral. Revista Brasileira de Plantas Medicinais, v.6, n.3, p.30-34, 2004.

HALL, C.A.; HOBBY, T.; CIPOLLINI, M. Efficacy and Mechanisms of $\alpha$-Solasonine- and $\alpha$-SolamargineInduced Cytolysis on Two Strains of Trypanosoma cruzi. Journal of Chemical Ecology. v.32, n.1, p.24052416, 2006.

HUNZIKER, A.T. The genera of Solanaceae. Ganther Verlag: Rugell. 79p. 2001.

KHAN, R.; ISLAM, B.; AKRAM, M.; SHAKIL, S.; AHMAD, A.A.; ALI, S.M.; SIDDIQUI, M.; KHAN, A.U. Antimicrobial Activity Of Fiver Herbal Extracts Against Muiti Drug Resistant (MRD) Strains Of Bacteria And Fungus Of Clinical Origin. Molecules, v.14, n.2, p.586-597, 2009

KIRAZ, N.U.; YASEMIN, O.Z. A distribuição das espécies e suscetibilidade in vitro de isolados clínicos de Candida de um hospital universitário na Turquia ao longo de um período de 5 anos. Medical Mycology, v.49, n.2, p.126-131, 2011.

LIMA, I.O.; OLIVEIRA, R.A.G.; LIMA, E.O.; FARIAS, N.M.P.; SOUZA, E.L. Atividade antifúngica de óleos essenciais sobre espécies de Candida. Revista Brasileira de Farmacognosia, v.16, n.2, p.197-201, 2006.

LUBIAN, C. T.; TEIXEIRA, J.M.; LUND, R.G.; NASCENTE, P.S.; DEL PINO, F.A.B. Atividade antifúngica do extrato aquoso de Arctiumminus (Hill) Bernh. (Asteraceae) sobre espécies orais de Candida. Revista Brasileira de Plantas Medicinais, v.12, n.2, p. 157-162, 2010.

MENDES, J. M. Investigação da atividade antifúngica de óleo essencial de Eugenia coryophyllata Thunb. Sobre cepas de Candida tropicalis. 2011. 78p. Dissertação (Mestrado em Produtos Naturais e Sintéticos Bioativos - Concentração: Farmacologia). Universidade Federal da Paraíba, João Pessoa, PB. 
NAKAMURA, S.; HONGO, M.; SUGIMOTO, S.; MATSUDA, H.; YOSHIKAWA, M. Steroidal Saponins and Pseudoalkaloid Oligoglycoside from Brazilian 22 Natural Medicine, "Fruta do Lobo" (fruit of Solanum lycocarpum). Phytochemistry, v.69, n.1, p.1565-1572, 2008.

NASCIMENTO, L.C.S.; SILVA, T.A.; ORLANDA, J.F.F. Atividade antimicrobiana in vitro de extratos vegetais de Solanum paniculatum L. sobre o crescimento de Ralstonia solanacearum. In: CONGRESSO BRASILEIRO DE QUÍMICA, 46, Anais... 2006. Salvador: ABQ, 2006. 12p.

NEE, M. Flora da Reserva Ducke, Amazonas, Brasil: Solanaceae. Rodriguésia, v.58, n.3, p.695-702, 2007.

NETO, M.F.; LIMA, R. A. Atividade Antifúngica do Extrato Etanólico dos Frutos de Solanum grandiflorum sobre Rhizoctonia solani in vitro. Revista Saúde e Pesquisa, v.7, n.1, p.103-108, 2014.

PIRES, L.S.S.; LIMA, R.A.; HERNÁNDEZ, A.E.F.; SOUZA, A.C.R.; SANTOS, M.R.A. Estudo fitoquímico do extrato etanólico das inflorescências de Solanum acanthodes Hook. MOSTRA CIENTÍFICA, 4, 2009, Porto Velho. Anais... Porto Velho: Ed. Faculdade São Lucas, 2009. 12p.

RIGO, V.A.A. Estudo fitoquímico preliminar do Solanum acanthodes Hook. (Família Solanaceae). 2008. 49p. Monografia de Graduação. Coordenação de Ciências Biológicas da Faculdade São Lucas, Porto Velho, 2008.

ROSA, R.L.; BARCELOS, A.L.V.; BAMPI, G. Investigação do uso de plantas medicinais no tratamento de indivíduos com diabetes melitos na cidade de Herval D' Oeste - SC. Revista Brasileira de Plantas Medicinais, v.14, n.2, p.306-310, 2012.

SANTOS, M. A. LIMA, R. A. Potencial Fungicida do Extrato das Folhas de Solanum acanthodes Hook. sobre Candida albicans in vitro. Revista Saúde e Pesquisa, v.6, n.3, p.373-378, 2013.

SILVA, T.M.S.; BATISTA, M.M.; CAMARA, C.A. \& AGRA, M.F. Molluscicidal Activity of Some Brazilian Solanum spp. (Solanaceae) Against Biomphalaria glabrata. Annals of Tropical Medicine \& Parasitology, v.99, n.4, p.419-425, 2005.

SILVA, T.M.S.; CAMARA, C.A.; FREIRE, K.R.L.; SILVA, T.G.; AGRA, M.F.; BHATTACHARYYA, J. Steroidal Glycoalkaloids and Molluscicidal Activity of Solanum asperum Rich. Fruits. Journal of Brazilian Chemical Society, v.19, n.5, p.1048-1052, 2008.

SILVA, A.C.; SALES, N.L.P; ARAÚJO, A.V; CALDEIRA-JÚNIOR, C.F. Efeito in vitro de compostos de plantas sobre o fungo Colletotrichum gloeosporioides Penz. isolado do maracujazeiro. Ciência e Agrotecnologia, v. 33, p. $1853-18602009$.

SOARES, E.L.C.; VIGNOLI-SILVA, M.; VENDRUSCOLO, G.S.; MENTZ, L.A. Solanaceae Nativas no Rio Grande do Sul, Brasil: Listagem I. Revista Brasileira de Biociências, v.5, n.2, p.1050-1052, 2007.

SOUZA, G.C.; HASS, A.P.S.; VON POSER, G.L.; SCHAPOVAL, E.E.S.; ELISABETSKY, E. Ethnopharmacological studies of antimicrobial remedies in the south of Brazil. Journal of Ethnopharmacology, v.90, n.1, p.135-143, 2004.

STOBIECKI, M.; MATYSIAK-KATA, I.; FRANSKI, R.; SKALA, J.; SZOPA, J. Monitoring changes in Anthocyanin and Steroid Alkaloid Glycoside Content in Lines of Transgenic Potato Plants Using Liquid Chromatography/Mass Spectrometry. Phytochemistry, v.62, n.1, p.959-969, 2003.

SUZUKI, L. C. Desenvolvimento de biofilme formado por Candida albicans in vitro para estudo da terapia fotodinâmica. 2009. 48f. Tese (Mestrado em Ciências na área de Tecnologia Nuclear - Materiais) Instituto de Pesquisas Energéticas Nucleares, Universidade de São Paulo, São Paulo, 2009. 
VALERI, B.; USUBILLAGA A. Sapogenins from Solanum meridense. Phytochemistry, v.28, n.9, p.2509-2511, 1989 .

VAZ, N.P. Constituintes Químicos de Solanum caavaurana Vell.: Isolamento, Mapeamento Fitoquímico por IES-EM/EM e sua aplicação no tratamento da Hanseníase. Tese de Doutorado, Universidade Federal do Paraná, 2010. 25p.

WEISSENBERG, M. Isolation of solasodine and other steroidal alkaloids and sapogenins by direct hydrolysis-extraction of Solanum plants or glycosides therefrom. Phytochemistry, v.58, n.1, p.501-508, 2001.

WINK, M. Evolution of secondary metabolites from an ecological and molecular phylogenetic perpective. Phytichemistry, v.64, n.1, p.3-19. 2003.

ZHOU, X.; HE, X.; WANG, G.; GAO, H.; ZHOU, G.; YE, W.; YAO, X. Steroidal saponins from Solanum nigrum. Journal of Natural Products, v.69, n.8, p.1158-1163, 2006. 